\title{
Surrogate insulin-producing cells
}

\section{Adrianne L. Wong, Albert Hwa, Dov Hellman and Julia L. Greenstein*}

\author{
Address: Juvenile Diabetes Research Foundation International, 26 Broadway, 14th Floor, New York, NY, 10005, USA \\ * Corresponding author: Julia L. Greenstein (jgreenstein@jdrf.org) \\ FI000 Medicine Reports 2012, 4:15 (doi:10.3410/M4-15) \\ This is an open-access article distributed under the terms of the Creative Commons Attribution-Non Commercial License \\ (http://creativecommons.org/licenses/by-nc/3.0/legalcode), which permits unrestricted use, distribution, and reproduction in any medium, \\ provided the original work is properly cited. You may not use this work for commercial purposes. \\ The electronic version of this article is the complete one and can be found at: http://f $1000 . c o m / r e p o r t s / m / 4 / 15$
}

\begin{abstract}
Diabetes, a large and growing worldwide health concern, affects the functional mass of the pancreatic beta cell, which in turn affects the glucose regulation of the body. Successful transplantation of cadaveric islets and pancreata for patients with uncontrolled type I diabetes has provided proof-ofconcept for the development of commercial cell therapy approaches to treat diabetes. Three broad issues must be addressed before surrogate insulin-producing cells can become a reality: the development of a surrogate beta-cell source, immunoprotection, and translation. Cell therapy for diabetes is a real possibility, but many questions remain; through the collaborative efforts of multiple stakeholders this may become a reality.
\end{abstract}

\section{Introduction}

Diabetes is a large and growing worldwide health concern currently affecting 285 million people worldwide and projected to reach 438 million by 2030. Diabetes complications, especially cardiovascular, are a major cause of morbidity, though obtaining exact statistics can be a challenge as many countries do not follow the disease. Almost four million deaths in the 20-79 age group may have been attributable to diabetes in 2010, accounting for $6.8 \%$ of global all-cause mortality in this age group. Estimated global healthcare expenditures to treat and prevent diabetes and its complications are expected to total at least US\$ 376 billion in 2010. By 2030, this number is projected to exceed some US $\$ 490$ billion [1].

Diabetes affects the functional mass of the pancreatic beta cell, which in turn affects the glucose regulation of the body. Diabetes is recognized as a group of heterogeneous disorders with the common elements of hyperglycemia and glucose intolerance, due to insulin deficiency, impaired effectiveness of insulin action, or both. In the progression of both type 1 (autoimmune, type 1 diabetes) and type 2 diabetes, there comes a point where a threshold percentage of the beta cells become dysfunctional, leading to the reliance on exogenous insulin to control blood sugar metabolism. An appealing scenario is to develop surrogate insulin-producing cells that can replace the damaged beta cells - and there is active interest in both academia and industry to develop the technology to do this. Successful transplantation of cadaveric islets and pancreata for patients with uncontrolled type 1 diabetes has provided proof-of-concept for the development of commercial cell therapy approaches. However, three main issues must be solved before surrogate insulin-producing cells can become a reality.

\section{Cell source}

Pancreas and pancreatic islet transplantation (from human cadaver pancreas) exist as clinical proof-ofprinciple for beta cell replacement therapy but have limitations of both quality and quantity. Fortunately, there are several potential surrogate insulin-producing cells that aim to overcome the limitations of cadaver sources. As the field lacks consensus on the definition of the attributes of a functional beta cell, it comes as no surprise that the identity of the "best" surrogate beta cell source has not been agreed upon by the field. Current contenders are xenogeneic (pig - currently in preclinical and clinical development) versus human pluripotent pancreatic progenitors (under preclinical development) versus human fully mature beta cells/islets (yet to be achieved). Other approaches are to reprogram or 
regenerate the few remaining beta cells in the body either in situ or ex vivo; the biology underlying these approaches is similar, though safety questions would differ.

\section{Immunoprotection}

Cadaveric islet transplantation utilizes an immunosuppression regime, which, along with the limited supply, keeps this approach from achieving more widespread adoption. The inevitable immune response will differ depending upon the nature of the transplanted surrogate cell source and the type of diabetes: the immune barrier is either allogeneic (between genetically different members of the same species) or xenogeneic (between different species), with the additional complication of autoimmunity in type 1 diabetes. This review will largely focus on a discussion of encapsulation technologies as an alternative strategy to protect surrogate beta cells, although there are approaches being tested using antigen-specific immune tolerance [2].

\section{Translation}

If the cell source is to be encapsulated, the proposed therapy will be a combination cell therapy and device product; both components must be optimized for function as a whole. The field is limited to a small number of large animal models for testing, whose relevance to how human subjects will react to these products remains questionable, and it is unclear what the regulatory agency requirements will be. Pluripotent stem cell-based therapies are still in their infancy, and there is very little precedent for safety testing. With these considerations, there has been much debate regarding the target patient population for early studies and for the proposed market.

\section{Current cell replacement therapy products and procedures}

In the past 3 decades, there have been major advances in research related to the replacement of islet cells destroyed by type 1 diabetes. Improvements in transplantation and immunosuppression management have resulted in the introduction of cadaveric pancreas and purified islet transplantation procedures. These procedures have demonstrated durable reversal of hypoglycemia unawareness (a frequent complication of diabetes therapy), reversal and/or stabilization of other diabetic complications and, in the case of whole pancreas transplants, induction of insulin independence for over 5 years in the majority of patients. At experienced centers using T cell depletion protocols, the insulin independence rate for islet transplantation is also approaching $50 \%$ at 5 years post-transplant. However, the availability of these treatments is generally limited to patients with severe diabetic symptoms (e.g. recurring hypoglycemia unawareness episodes) or those already receiving a kidney transplantation. As a result, the number of both pancreas and islet transplant procedures has been restricted to around $1,100-1,500$ over the past few years $[3,4]$.

The relative limited availability of these procedures is due to a number of factors including the following: 1) a severe shortage of suitable cadaveric donor pancreata according to the National Institute of Diabetes and Digestive and Kidney Diseases (NIDDK), organs from about 7,000 deceased donors become available each year in the U.S. and fewer than half of the donated pancreata are suitable for whole organ pancreas transplantation or for harvesting of islets [5]; 2) surgical risks - which are more substantial for whole pancreas transplants; and 3) requirement of lifelong immunosuppression, which can have serious side effects and severely limits the procedures to a small subset of the type 1 diabetes population. Finally, the significant costs associated with preparing islet isolations and procuring cadaveric pancreata have dramatically slowed down islet transplants. Due to very different regulation rules on cell transplantation in different countries, these costs are either largely paid for by national health services (e.g. Canada, UK, and several European countries) or are solely supported by research grant funds (i.e. USA). The organ costs in the U.S. are particularly prohibitive for transplant centers to perform a large number of islet transplants owing to the procurement costs for pancreases that are later found not to yield transplantable islets [6].

\section{Cell source}

A robust source of mature islets could significantly decrease the number of islets required for inducing insulin independence. A well-manufactured source of beta cells will have much higher quality control than is possible for cadaveric islet isolations, such that beta cell survival, longevity and function after transplantation will be improved. This may be a particularly sensitive issue for encapsulated cells, where a non-vascularized environment can prove unfavorable for the engraftment of newly transplanted cells. Most importantly, there would be an increased supply of islet cells available to patients compared to just cadaveric islets accessible today. There are three major cell source categories currently under development: 1) xenogeneic islets - using islets isolated from "clean" defined pathogen-free pigs (porcine [pig] islets have become the dominant choice as a source species for xenotransplantation because of their physiologic similarity to human islets, the demonstrated clinical efficacy of porcine insulin, and the favorable breeding characteristics and biology of the pig); 2) stem cells - growing stem cells, primarily human embryonic stem cells (hESC) or induced pluripotent stem cells (iPSC), scaling them up and differentiating them into a 
commercially relevant supply of pancreatic progenitor or functional islets/beta cells; and 3) reprogramming (trans-differentiation) adult non-beta cells by gene therapy or other approaches to become functional "surrogate beta-cells" glucose-responsive, insulin-secreting cells, with the potential advantage of evading the autoimmune attack in type 1 diabetes [7].

\section{Xenogeneic islets}

Studies published in 2006 provided the proof-of-concept that porcine islets can restore beta cell function in a nonhuman primate model (albeit with heavy immunosuppression) $[8,9]$. There is on-going preclinical research and development to achieve the same result with clinically applicable immunosuppression protocols. Notably, Living Cell Technologies is currently conducting phase IIb trials for its alginate-encapsulated porcine islet product DIABECELL in New Zealand and Argentina. An attractive feature of xenogeneic islets is the potential for genetic engineering to remove or express transplantfriendly molecules on the target tissue. Such a strategy would focus on removal of the galactose sugar against which humans have natural antibodies, secretion and/or surface expression of immunomodulatory molecules, and interfering with other innate and adaptive immunity pathways. In fact, the GalT-Knockout pig strain is currently being used to generate pigs with more than one such modification. It is therefore expected that, in the near future, there will be multiple clinical trials to test porcine islets as treatments for type 1 diabetes with various formulations and genetic makeups, with encapsulation and/or immunomodulatory drugs.

\section{Human stem-cell derived islets}

There is some debate as to whether the optimal commercial cell therapy product would embody the following: 1) a fully mature transplantable beta cell population; 2) a fully mature islet population; or 3) a pancreatic progenitor cell population (Figure 1). At present, the state of the art allows testing of only number 3), an in vitro differentiation protocol that results in a pancreatic progenitor cell population which, once transplanted into a mouse and matured over three months, can provide physiologic glucose control to the animal [10].

These pancreatic progenitor cells were only made on a small scale, so optimization, standardization, scale-up, and transition to good manufacturing practice must all be performed before they could become clinically useful. Further, independent replication of various published in vitro pancreatic lineage differentiation protocols has demonstrated the importance of the starting cell line for successful differentiation. For example, the Viacyte protocol has been demonstrated to be most effective with the Cyt203 line [11] but if this line is not acceptable to the FDA, the protocol must be optimized with a new cell line. Potential products 1) and 2) currently lack the basic biological understanding of how to form a mature and functional beta cell/islet in vitro. This could be achieved by improving the later stages of the current state-of-the-art protocols or by attempting to alter steps earlier in the differentiation protocol in an attempt to confer the capacity to fully mature these cells. In addition, the ability to recapitulate the in vivo "niche" for functional maturation of progenitors through cellular co-culture or engineering cell scaffolds is still under investigation [12].

\section{Immunoprotection}

Regardless of the cell source of choice, some form of protection from allogeneic, xenogeneic, and autoimmune responses is needed to ensure long-term health and function of the transplanted cells. Clearly, the (yet to be achieved) Holy Grail of specific induction of transplant tolerance would be the ideal solution. Meanwhile, it has been recognized that cell encapsulation is a unique technology for immunoprotection to allow beta cell replacement $[13,14]$. A successful encapsulation technology would increase the access of cell replacement therapy to a broader patient base by eliminating/ minimizing the need for chronic administration of immunosuppressive drugs. Furthermore, the use of a macro-encapsulation device will enable retrievability of the entire graft, which may be a desirable feature for products using hESC-derived cells as a potential safety benefit, allowing removal of the cells if there are complications with them.

Encapsulation technologies use immune-protective biomaterials to create a permselective membrane around transplanted cells and are thereby designed to limit and ideally eliminate undesirable immunological responses to the non-host islet cells. A permselective biocompatible material allows for small molecule diffusion of oxygen, glucose, insulin and other nutrients, while blocking larger molecules, such as immune cells and antibodies. This strategy will ideally protect cells entirely from autoimmune attack. However, a permselective membrane is, in most cases, selective based on pore size. It may not be possible to reduce the pore size to completely eliminate the passage of cytokines or even smaller molecules such as nitric oxide without significantly impeding the transport of insulin and other cell survival factors; it is unclear if cytokines will be toxic or if the immune system will be activated by encapsulated cells. The design of a successful encapsulation membrane or device will likely need to be extremely biocompatible to 
Figure I. General considerations for the development of hESC based replacement cell sources

\section{Human Embryonic Stem Cell-Derived Beta Cells}

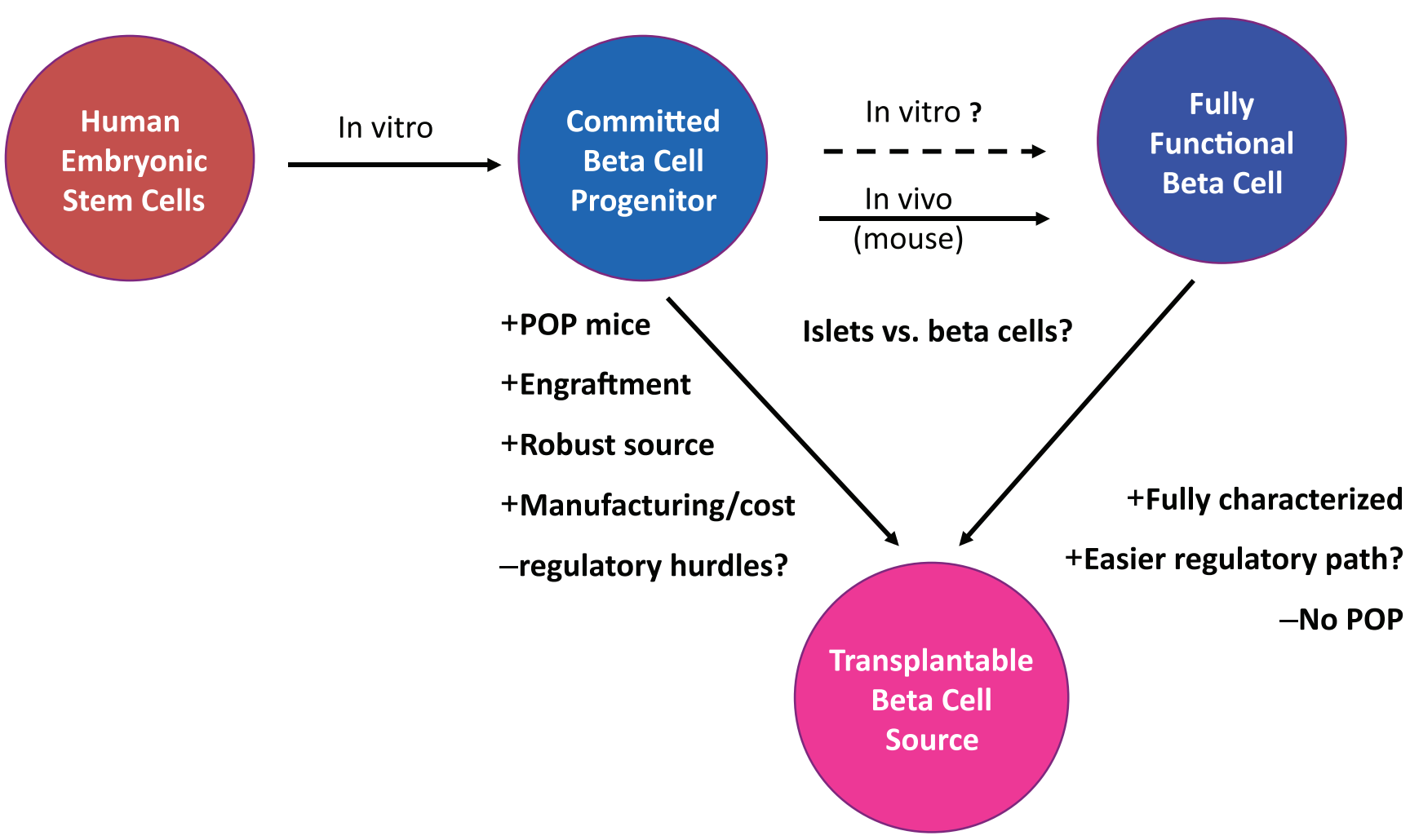

As depicted, protocols have been developed to differentiate hESC in vitro to committed beta cell progenitors; one protocol has resulted in pancreatic progenitors that can be matured in vivo when transplanted into mice into fully functional beta cells. However, it remains to be seen whether it will be possible to develop protocols to make fully differentiated, functional beta cells in vitro. Consideration of whether to develop a committed beta cell progenitor (A) or a fully functional beta cell (B) for clinical proof of concept and potential commercialization include the following analyses: A has the benefit of Proof of Principle (POP) for maturation in mice to functional beta cells, potential advantages for engraftment and robustness, and the potential for cost savings with a shorter, simpler cell product manufacturing procedure, while there may be greater regulatory hurdles with a progenitor cell product in the form of regulatory safety and characterization hurdles. B may have a somewhat easier potential regulatory path in that the cell product need not be further matured post transplantation; however there is currently no published proof of principle that these cells might be differentiated in vitro.

minimize attraction of immune cells and the occurrence of cell-damaging molecules. It may also require additional biologically active molecules to create an anti-inflammatory and immune regulatory environment that is hospitable to the transplanted cells. It is also possible that the requirements for the encapsulation system will differ depending on the cell source and implantation site chosen. Devices under investigation differ by biomaterials, physical configuration, and methods used in fabrication. Several materials are, or have been, used to encapsulate islets including alginate, agarose, polysulphone, and polyethylene glycol (PEG). Encapsulation schemes can be broadly categorized into macro-encapsulation devices - one device containing a large mass of cells, and micro-capsules - each capsule containing single islets or small groups of islets. Newer micro-encapsulation technologies under development aim to greatly reduce the thickness of the capsule wall: conformal coating uses a novel co-axial flow apparatus to achieve uniform but thin coverage of islets; nano-encapsulation typically uses chemical and electrostatic interactions to deposit biomaterials layer-by-layer at the nanometer scale.

Micro- and macro-encapsulation technologies offer different advantages/disadvantages. The distance between encapsulated cells from the nearest blood supply poses a limitation on cell survival and proper glucose regulation, and this is usually a larger factor for macro-encapsulation. 
Table I. Immunoprotection strategies - Summary of the advantages and disadvantages of three broad categories of immunoprotection strategies for cell replacement for type I diabetes: unencapsulated cell therapy, microencapsulation, and macroencapsulation

\begin{tabular}{|c|c|c|c|}
\hline & Unencapsulated & Microencapsulation & Macroencapsulation \\
\hline Advantages & $\begin{array}{l}\text { Anatomical integration between } \\
\text { host and transplanted islet. }\end{array}$ & $\begin{array}{l}\text { Use of allo- and xeno-islets with- } \\
\text { out immunosuppression. } \\
\text { Thin wall and spherical shape are } \\
\text { optimal for cell viability and faster } \\
\text { diffusion of nutrients and insulin. }\end{array}$ & $\begin{array}{l}\text { Use of allo- and xeno-islets without immunosuppression. } \\
\text { Good mechanical stability. } \\
\text { Good cell viability and faster diffusion of nutrients and } \\
\text { insulin. } \\
\text { Retrievable. }\end{array}$ \\
\hline Disadvantages & $\begin{array}{l}\text { Requires chronic immuno- } \\
\text { suppression or tolerance strategy. } \\
\text { Limited retrievability. }\end{array}$ & $\begin{array}{l}\text { Mechanically and chemically fragile. } \\
\text { Limited retrievability. }\end{array}$ & $\begin{array}{l}\text { Internal characteristics (i.e. diameter or depth) may } \\
\text { potentially limit free diffusion of nutrients, insulin, etc. in } \\
\text { turn negatively affecting insulin release kinetics and cell } \\
\text { viability. } \\
\text { Need for multiple implants may produce significant tissue } \\
\text { displacement/damage. }\end{array}$ \\
\hline
\end{tabular}

Adapted from [15]

Free floating micro-capsules, on the other hand, do not allow complete graft retrieval. Table $1[15]$ illustrates the potential advantages/disadvantages of transplantation of unencapsulated, micro-encapsulated, and macroencapsulated islets. Currently, the encapsulation field has been successful at protecting islets from allo-immunity in rodent models. Translating these results into large animal models has been a bottleneck. Furthermore, mechanistic studies on how to improve long-term islet function will be necessary to generate better future encapsulation designs.

\section{Translation}

\section{Preclinical testing}

The identity of the cell source under development will determine the path of preclinical safety and efficacy testing. Specifically, xenogeneic islets will have different safety and efficacy concerns than human stem cellderived progenitor, beta, or islet cells [16]. Early discussions with the appropriate regulatory authorities will be useful to gain insight to their potential requirements for large animal studies, if any. While a number of models are useful for various aspects of type 1 diabetes and type 2 diabetes, there is no one model the field has come to agreement on as the gold standard.

\section{Stem cell clinical translation}

There have been proposals to use stem cells in the clinic $[16,17]$, and the National Institutes of Health and the Food and Drug Administration are collaborating on a series of workshops on moving pluripotent stem cell therapies into the clinic, the first of which was in March 2011 [18]. The second focused on preclinical considerations and was in June 2012.

Tumorigenesis is the first safety concern that comes to mind in relation to stem cell-derived cell replacement. If cell replacement will be achieved by in situ differentiation of transplanted progenitor cells, the impetus will be on the applicant to prove that these cells will only be capable of differentiation into the desired therapeutic cell type and lack the capacity to follow another pathway or de-differentiate. Further, quality control and release criteria must be devised to reproducibly characterize progenitor cells and their capacity to mature into functional glucose-responsive insulin producing beta cells, which may be difficult as the field currently lacks established methods to assess the intermediate stages of beta cell development [19]. For any cell replacement source, whether beta cells "terminally differentiated" in vitro or pancreatic progenitors, a group would need to prove that the proposed cell therapy lacked a significant population of pluripotent, undifferentiated cells. What percentage would be viewed as "significant" also remains to be established. The source of hESC is another issue: hESC are notoriously finicky on the best day, and it has been well established that different hESC lines have varying capacity for differentiation along certain lineages. The development of a master current good manufacturing practice bank of a line that consistently succeeds at making pancreatic progenitors or beta cells, scaled to human therapeutic quantities, and allows commercial storage and transport of these cells will be a significant undertaking.

\section{Combination products}

As discussed previously, encapsulation is an approach to protect replacement beta cells from the auto- and alloimmune attack. In the US, a potential product combining replacement cells (biological product) and encapsulation (device) would be considered a "combination product"; the regulatory pathway for clinical approval would require input from both CDRH (Center for Device and Radiological Health) and CBER (Center for Biologics Evaluation and Research)/OCTGT (Office of Cellular, Tissue, and Gene Therapy), though CBER will take the 
lead [20]. There is no precedent for such a combination product, so the first company or investigator will be a trailblazer.

\section{Target patient population}

A key aspect of the clinical study design and downstream commercialization efforts will be identifying the optimal target patient population for surrogate replacement cell therapy. As insulin therapy represents a well-established, safe therapeutic modality for diabetes, which is a chronic disease, the risk-benefit ratio bar will be set quite high for a cell therapy with immunosuppression in the general population of individuals currently using insulin therapy. Clearly, given this scenario, the elimination of the need for immunosuppression would potentially tip the balance in favour of cell therapy. The patient population that would benefit the most would be "brittle" diabetics with poor control and hypoglycemic unawareness. From the perspective of optimizing conditions for surrogate beta cell graft success, however, transplantation into a patient with poor control would put immediate demands on the transplant, which might lead to beta cell stress and less than optimal engraftment. Conversely, for early phase I/II studies it is unclear whether it would be appropriate to study cell transplant efficacy and safety in an otherwise well-controlled and healthy person with diabetes. Other situations such as pancreatectomy patients are under consideration, and this decision will most certainly be made with the input of scientists, clinicians, and patient advocates.

\section{Companies and commercialization}

Industry research efforts into encapsulated cell sources began to surface as early as four decades ago and, by some estimates, have totalled more than US\$ 600 million. However, many efforts within the industry have been abandoned, or passed on to other companies, and research to date has yet to be proven to date to translate the underlying science into commercially successful products. Today, only a few companies are active; only Living Cell Technologies in New Zealand has a clinical program and four companies have committed significant resources to the development of a cell source for diabetes and its translation.

Since the end-product of an encapsulated cell therapy will include a viable cell source and an encapsulation technology, industry players may be segmented into: 1) companies developing an encapsulation technology and a scalable cell source; 2) companies solely focused on developing an encapsulation technology; and 3) companies solely focused on developing a scalable cell source. The latter two categories could potentially partner to offer an encapsulated cell therapy product. (Table 2)

\section{Economics and practicalities Porcine cell source}

Currently, medical grade designated pathogen-free pig herds are being raised for research purposes only. These herds are bred and maintained in bio-secure facilities, where they are shielded from the outside by filtered air, disinfected water and irradiated food that is certified free of any mammalian protein.

Scaling up pathogen-free pig herds to meet the potential addressable patient market will likely take time and require considerable resources. According to the International Xenotransplantation Association [21], islet mass sufficient for restoring euglycemia $(>10,000$ islet equivalents $/ \mathrm{kg}$ ) would likely require pooling of islets from multiple donor pancreata (the number of pigs required to treat one patient depends on the patient's weight and required dose). International Xenotransplantation Association estimates the required number of pig donor pancreata per patient ranges from two to four from adult donors and seven to ten from neonatal donors. However, these are likely upper estimates as human physiology may require fewer islets compared to non-human primates. Further, there may also be room for improvement on tissue processing and neonatal tissue expansion. Nonetheless, before a porcine cell source-derived product enters the market, production would need to be substantially scaled from the hundreds of pathogen-free pigs available for research today. Establishing a bio-secure facility is expensive-Spring Point Project spent over US\$ 6 million constructing its 21,000 square-foot facility (with a current capacity of 100 medical-grade pigs) in western Wisconsin (similarly, Living Cell Technologies paid around $\$ 2.5$ million to establish a facility for a capacity of 50 pigs in Invercargill, New Zealand in 2009). Moreover, due to the breeding characteristics of pigs (according to our estimates, a sow becomes fertile at 6 months of age and pregnancy takes over 3 months) and the required safety controls, raising a herd for commercial scale can take a number of years. For example, Living Cell Technologies, which focuses on a neonatal pig donor cell source, estimates that it could take 3 years to grow a herd from 100 sows to 36,000 piglets.

These factors and the related procedure expenses/ requirements could drive up the overall cost per treatment. Beckwith et al. [22] performed a health economic analysis of porcine islet xenotransplantation. They estimated the cost of source islets from young pigs ranges from US $\$ 5,000-\$ 10,000$. Adding islet manufacturing and other related expenses (including safety monitoring and the medical procedure) resulted in an estimated procedure cost of US\$58,000 to $\$ 118,000$ depending on the number of pig donors required for the 


\section{Encapsulation/Cell Source Industry Landscape}

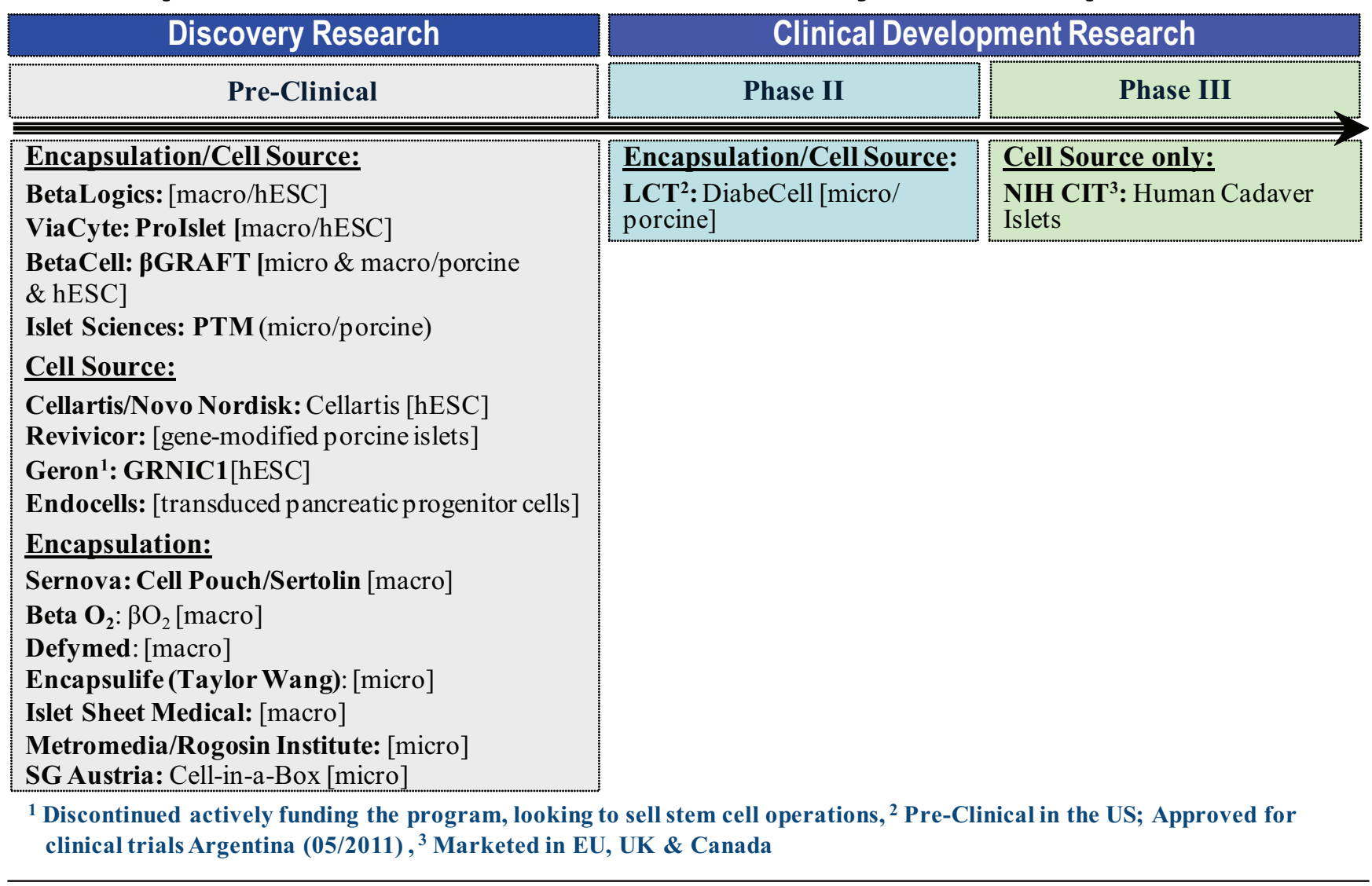

procedure. Despite the high initial costs, however, porcine islet transplantation with immunosuppression was shown to be a theoretically more cost effective therapy for type 1 diabetes than standard insulin therapy. Assuming an encapsulation technology would eliminate immunosuppression (a cost of US\$30,000 per year), the relative cost effectiveness for an encapsulated therapy would be even better.

\section{Stem cell source}

Details surrounding the development and scale up of stem cell-derived cell sources remain uncertain compared with porcine islets. There is no precedent for the use of replacement islet/stem cell therapy in patients with diabetes and there is limited precedent for non-autologous stem cell therapy overall. Much of the work to date on the development of stem cell therapy treatments for type 1 diabetes patients has been performed using mouse models and, to a more limited extent, in non human primates. Therefore, there are still many unknowns related to the required treatment dosing (islet equivalents), required safety/efficacy measures, and production/scale up/cryopreservation process and costs.

Development efforts focus primarily on hESC- and iPSCderived cell sources and apply different differentiation protocols, requiring a multi-stage production process. A current published protocol from ViaCyte for a hESCderived cell source starts with pluripotent cells (Cyt203) and differentiates them into pancreatic progenitors (pancreatic endoderm cells), which are then implanted into mice and allowed to mature in vivo. The protocol has been optimized for ViaCyte's current good manufacturing practice hESC cell line and has been shown in mouse models to produce islet equivalent cells with many of the properties of functional beta cells. The production scale up is expected to be achieved by expanding hESCs, then going through a step-wise differentiation in a controlled environment using a bioreactor yielding the pancreatic progenitor cells. Finally, they will be cryopreserved for future use and thawed immediately before implantation. It should be noted that there are strong supporters of the 
continued preclinical development of a fully mature, functional beta-cell/islet source as an ideal therapeutic; many academic groups and at least one pharmaceutical company are currently pursuing this line of investigation.

While the ViaCyte protocol and production process sounds promising, it is still under development and has yet to be tested in primates or people. This is also true for other companies developing a stem cell-derived source of islets/beta cells. Therefore, there are still many potential bottlenecks and unknowns surrounding the commercial production of a finalized stem cell-derived cell source, whether progenitor or fully mature, thereby further complicating any economic assessment at the current stage of development.

\section{Future prospects}

\section{What does the future hold?}

Currently, there is still a need for basic studies to unlock the secrets to beta cell maturation; most of this work is taking place in academia with the close attention of companies in this area. Application of these studies to the optimization and scale-up of a cell-based product will be useful, though companies moving the first product into the clinic will be required to put aside explorations of further biological questions in favor of product development. The decision between pancreatic progenitors, betacells, and islets as the surrogate beta cell of choice will be dependent upon both the science available and the practicalities of getting a product to the market to treat patients. Optimization of the cell source must take place in parallel with the development of technologies to protect the transplanted graft: novel encapsulation materials and design for immunoprotection without immunosuppression are thought to be essential for the commercialization of this therapeutic approach.

\section{What questions remain?}

The key scientific questions for the cell source are what factors control the maturation of beta cells in vivo and how these can be recapitulated in vitro. There remains much to be done in encapsulation research - development of novel biomaterials, robust xenogeneic protection in a large animal model, and elucidating the keys to long-term encapsulated islet health and function. For the first generation commercialization, the field must decide whether to develop a progenitor versus fully mature beta cell/islet source and how best to protect cell source, and define the patient population for first generation therapeutic trials. Cell therapy for diabetes is a real possibility; it is only through the collaborative efforts of multiple stakeholders that this can become a reality.

\section{Abbreviations}

hESC, human Embryonic Stem Cell; iPSC, induced Pluripotent Cell; FDA Food and Drug Administration

\section{Competing interests}

"The author(s) declare that they have no competing interests."

\section{References}

I. International Diabetes Federation. IDF Diabetes Atlas, 4th edn. Brussels, Belgium: International Diabetes Federation, 2009.

2. Luo X, Herold KC, Miller SD: Immunotherapy of type I diabetes: where are we and where should we be going? Immunity 2010, 32:488-99.

\section{FI000 Factor 6}

Julia Greenstein 20 Jul 2012

3. Collaborative Islet Transplant Registry website [http://www. citregistry.org/]

4. The Organ Procurement and Transplantation Network [http://optn. transplant.hrsa.gov/]

5. National Diabetes Information Clearinghouse [http://diabetes.niddk. nih.gov/dm/pubs/pancreaticislet/]

6. Markmann JF, Kaufman DB, Ricordi C, Schwab PM, Stock PG: Financial issues constraining the use of pancreata recovered for islet transplantation: a white paper. Am J Transplant 2008, 8: 1588-92.

FI000 Factor 6

Julia Greenstein 20 Jul 2012

7. Tudurí E, Kieffer TJ: Reprogramming gut and pancreas endocrine cells to treat diabetes. Diabetes Obes Metab 201I, I3(Suppl I):53-9.

FI000 Factor 6

Julia Greenstein 20 Jul 2012

8. Hering $B J$, Wijkstrom $M$, Graham ML, Hårdstedt $M$, Aasheim TC Jie T, Ansite JD, Nakano M, Cheng J, Li W, Moran K, Christians U, Finnegan C, Mills CD, Sutherland DE, Bansal-Pakala P, Murtaugh MP, Kirchhof N, Schuurman H: Prolonged diabetes reversal after intraportal xenotransplantation of wild-type porcine islets in immunosuppressed nonhuman primates. Nat Med 2006, 12:30|-3.

FI000 Factor 6

Julia Greenstein 20 Jul 2012

9. Cardona K, Korbutt GS, Milas Z, Lyon J, Cano J, Jiang W, Bello-Laborn H, Hacquoil B, Strobert E, Gangappa S, Weber CJ, Pearson TC, Rajotte RV, Larsen CP: Long-term survival of neonatal porcine islets in nonhuman primates by targeting costimulation pathways. Nat Med 2006, I 2:304-6.

FI000 Factor 6

Julia Greenstein 20 Jul 2012

10. Kroon E, Martinson LA, Kadoya K, Bang AG, Kelly OG, Eliazer S, Young H, Richardson M, Smart NG, Cunningham J, Agulnick AD, D'Amour KA, Carpenter MK, Baetge EE: Pancreatic endoderm derived from human embryonic stem cells generates glucose-responsive insulin-secreting cells in vivo. Nat Biotechnol 2008, 26:443-52.

FI000 Factor 8

Bart Roep 31 Mar 2008, Kenneth Zaret 14 May 2008, Julia Greenstein 20 Jul 2012 
II. D'Amour KA, Bang AG, Eliazer S, Kelly OG, Agulnick AD, Smart NG, Moorman MA, Kroon E, Carpenter MK, Baetge EE: Production of pancreatic hormone-expressing endocrine cells from human embryonic stem cells. Nat Biotechnol 2006, 24: $1392-40 \mid$.

FI000 Factor 18

Kenneth Zaret 26 Oct 2006, Stephen Dalton 26 Oct 2006 Raghavendra Mirmira 16 Nov 2006, Victoria Prince 14 Dec 2006, Julia Greenstein 20 Jul 2012

12. Seymour PA, Sander M: Historical perspective: beginnings of the beta-cell: current perspectives in beta-cell development. Diabetes 20II, 60:364-76.

FI000 Factor 6

Julia Greenstein 20 Jul 2012

13. Korsgren $\mathrm{O}$, Nilsson B: Improving islet transplantation: a road map for a widespread application for the cure of persons with type I diabetes. Curr Opin Organ Transplant 2009, I 4:683-7.

FI000 Factor 6

Julia Greenstein 20 Jul 2012

14. Kiessling FM: Science to practice: are theranostic agents with encapsulated cells the key for diabetes therapy? Radiology 201 I, 260:613-5.

FI000 Factor 6

Julia Greenstein 20 Jul 2012
I5. Soria B, Hmadcha A, Bedoya F, Tejedo J: Generation of Islets from Stem Cells. In Principals of Tissue Engineering 3rd edition. Edited by Lanza R, Langer R, Vacanti J. Burlington: Elsevier; 2007, 605-I5.

16. CIRM/Regenerative Medicine Consortium Webinar: Preclinical Considerations for Stem Cell Therapies [http://www.cirm.ca.gov/agenda 09-28-10_Webinar]

17. Wong AL, Nierras CR: Do stem cell-derived islets represent a commercially viable treatment for Type I and 2 diabetes? Regen Med 2010, 5:839-42.

18. NIH-FDA Workshop: Pluripotent Stem Cells in Translation: Early Decisions [http://www.ninds.nih.gov/news_and_events/proceedings/ nih-fda_workshop.htm http://videocast.nih.gov/summary.asp?live=10013 http://videocast.nih.gov/summary.asp?live $=1008 \mathrm{I}]$

19. Next Generation Beta-Cell Transplantation: How good does a beta cell have to be? November 9, 2009 [http://www3.niddk.nih.gov/fund/ other/nextgenbeta/agenda.htm]

20. Food and Drug Administration: Vaccines, Blood \& Biologics [http:// www.fda.gov/BiologicsBloodVaccines/default.htm]

21. The International Xenotransplantation Association Consensus Statement on Conditions for Undertaking Clinical Trials of Porcine Islet Products in Type I Diabetes.

22. Beckwith J, Nyman JA, Flanagan B, Schrover R, Schuurman $H$ : A health-economic analysis of porcine islet xenotransplantation. Xenotransplantation 2010, 17:233-42.

FI000 Factor 6

Julia Greenstein 20 Jul 2012 